\title{
Multi-baryon systems
}

\author{
Kostas Orginos ${ }^{* \dagger}$ \\ College of William and Mary / JLab \\ E-mail: kostas@wm. edu
}

\section{William Detmold \\ $C T P / L N S, M I T$ \\ E-mail: wdetmoldemit.edu}

In this talk I review the challenges related to calculations of properties of interacting baryons on the lattice. I present the progress made addressing the problem of calculating the large number of Wick contractions necessary to compute states with the quantum numbers of many baryons in lattice QCD. Examples of correlation functions computed using these techniques are shown for the quantum numbers of the light nuclei, ${ }^{4} \mathrm{He},{ }^{8} \mathrm{Be},{ }^{12} \mathrm{C},{ }^{16} \mathrm{O}$ and ${ }^{28} \mathrm{Si}$.

The 30th International Symposium on Lattice Field Theory

June 24 âĂS 29, 2012

Cairns, Australia

* Speaker.

${ }^{\dagger}$ We acknowledge computational support from the National Energy Research Scientific Computing Center (NERSC, Office of Science of the US DOE, DE-AC02-05CH11231), and the NSF through XSEDE resources provided by NICS. This work was supported in part by DOE grants DE-AC05-06OR23177 (JSA) and DE-FG02-04ER41302.

${ }^{\ddagger}$ WD was supported by DOE OJI grant DE-SC0001784 and Jeffress Memorial Trust, grant J-968. 


\section{Introduction}

The calculation of properties of multi-baryon systems in lattice QCD is in many aspects a very challenging problem. However, several lattice calculations have recently been performed indicating that certain properties are calculable with current resources and methodologies $[1,2,3,4,5,6,7,8]$. The many body nature of this problem seem to be at the core of the difficulties encountered in present works. Both the construction of relevant interpolating fields and correlation functions, as well as the Monte-Carlo evaluation of the path integral that defines them are quite challenging.

Here we present a systematic method for the construction of nuclear interpolating fields for multi-baryon systems in lattice QCD (LQCD) (see Ref. [9] for related work). In addition, we present an approach for performing the relevant contractions which scales only polynomially in the number of quarks involved in the contraction. Using these methods we compute LQCD correlation functions with the quantum numbers of the light nuclei, ${ }^{4} \mathrm{He},{ }^{8} \mathrm{Be},{ }^{12} \mathrm{C},{ }^{16} \mathrm{O}$ and ${ }^{28} \mathrm{Si}$, demonstrating that correlation functions relevant to the study of nuclei in QCD can be constructed. A more detailed presentation of this work can be found at [10].

\section{Nuclear Interpolating fields}

In order to calculate nuclear correlation functions, we first need to construct quark level nuclear interpolating fields. This is in principle straightforward and in practice it resembles the construction of quark model wave-functions for baryons [11]. A general quark-level nuclear interpolating field with atomic number $A$ containing $n_{q}=3 A$ quarks has the form

$$
\overline{\mathscr{N}}^{h}=\sum_{\mathbf{a}} w_{h}^{a_{1}, a_{2} \cdots a_{n_{q}}} \bar{q}\left(a_{1}\right) \bar{q}\left(a_{2}\right) \cdots \bar{q}\left(a_{n_{q}}\right),
$$

where the $\bar{q}_{a_{i}}$ are the quark fields, the $a_{i}$ are generic indices which combine the colour, spinor, flavour, and spatial indices of the quark and $\mathbf{a}$ is a compound index representing the $n_{q}$-plet $a_{1}, a_{2} \cdots a_{n_{q}}$. Given that calculations are performed on a discrete lattice, the spatial degrees of freedom are finite and countable, and as a result we can use an integer index to describe them. Here the quark fields are all at the same time $t$. The index $h$ on the nuclear interpolating field is a set of quantum numbers that identify the nuclear state, including its momentum, angular momentum, isospin and strangeness. The Grassmannian nature of the quark field dictates that the tensor $w_{h}^{a_{1}, a_{2} \cdots a_{n q}}$ is totally antisymmetric under the exchange of any two indices. If the indices $a_{i}$ can have a total of $N$ possible values, then, ignoring the detailed flavour structure, the total number of non-vanishing terms, taking into account Grassmanian interchange symmetry, in the above sum is $\frac{N !}{n_{q} !\left(N-n_{q}\right) !}$. The first major reduction to the above number of terms comes from the fact that only colour singlets need to be considered. In addition, considering only interpolating fields of definite parity, angular momentum ${ }^{1}$, isospin and strangeness, forces several elements of the tensor $w_{h}^{a_{1}, a_{2} \cdots a_{n_{q}}}$ to vanish. Finally, the most drastic reduction of the non-zero tensor elements can be achieved using simple spatial wave-functions. At this time, having recognised that only a small fraction of the terms in the sum of Eq. 2.1 are non-zero, as well as the fact that the tensor $w_{h}^{a_{1}, a_{2} \cdots a_{n_{q}}}$ is totally anti-symmetric, we can introduce the reduced weights $\tilde{w}_{h}^{\left(a_{1}, a_{2} \cdots a_{n_{q}}\right), k}$ which are the minimal set of non-zero numbers

\footnotetext{
${ }^{1}$ For simplicity, we refer to the irreducible representation of the lattice symmetry group as angular momentum.
} 
required to completely describe the interpolating field. The $n_{q}$-plet $\left(a_{1}, a_{2} \cdots a_{n_{q}}\right)$, is an ordered list of indices that represents a class of terms in Eq. 2.1 that are all permutations of each other. The index $k$ on the reduced weights enumerates the number of classes that the tensor $w_{h}^{a_{1}, a_{2} \cdots a_{n_{q}}}$ decomposes into. With these reduced weights, Eq. 2.1 can be re-written as

$$
\overline{\mathscr{N}}^{h}=\sum_{k=1}^{N_{w}} \tilde{w}_{h}^{\left(a_{1}, a_{2} \cdots a_{n_{q}}\right), k} \sum_{\mathbf{i}} \varepsilon^{i_{1}, i_{2}, \cdots, i_{n_{q}}} \bar{q}\left(a_{i_{1}}\right) \bar{q}\left(a_{i_{2}}\right) \cdots \bar{q}\left(a_{i_{n_{q}}}\right),
$$

where $N_{w}$ is the total number of reduced weights, $\mathbf{i}$ represents the $n_{q}$-plet $\left(i_{1}, i_{2} \cdots i_{n_{q}}\right)$ and $\varepsilon^{i_{1}, i_{2}, \cdots, i_{n_{q}}}$ is a totally anti-symmetric tensor of rank $n_{q}$ with $\varepsilon^{1,2,3,4, \cdots, n_{q}}=1$. The above expression is the simplest form of the quark-level nuclear interpolating field and is completely described by the reduced weights. Using a single point spatial wave function, the numbers of terms contained in the interpolating fields for the proton, deuteron, ${ }^{3} \mathrm{He}$ and ${ }^{4} \mathrm{He}$, are $N_{w}=9,21,9$, and 1, respectively.

Having now written down a general nuclear interpolating field with quantum numbers $h$, we need to calculate the reduced weights $\tilde{w}_{h}^{\left(a_{1}, a_{2} \cdots a_{n q}\right), k}$ in an efficient manner. In principle, this can be achieved directly from quark fields by imposing the desired transformation properties. However, in certain cases, it is advantageous to proceed by first constructing hadronic interpolating fields from which the quark interpolating fields are derived. The hadronic interpolating fields assume a form analogous to that of the quark interpolating fields. The baryons that make up the nucleus are also fermions, hence the general structure outlined above can be directly transcribed here. In terms of baryons, a nuclear interpolating field of a nucleus of atomic number $A$ is

$$
\overline{\mathscr{N}}^{h}=\sum_{k=1}^{M_{w}} \tilde{W}_{h}^{\left(b_{1}, b_{2} \cdots b_{A}\right)} \sum_{\mathbf{i}} \varepsilon^{i_{1}, i_{2}, \cdots, i_{A}} \bar{B}\left(b_{i_{1}}\right) \bar{B}\left(b_{i_{2}}\right) \cdots \bar{B}\left(b_{i_{A}}\right),
$$

where $M_{w}$ is the number of hadronic reduced weights $\tilde{W}_{h}^{\left(b_{1}, b_{2} \cdots b_{A}\right)}, B\left(b_{i}\right)$ are baryon interpolating fields and the $b_{i}$ are generic indices that includes parity, angular momentum, isospin, strangeness, and spatial indices. Unlike the quark fields which are fundamental degrees of freedom, the baryon interpolating fields are composite objects, hence there is a large number of such interpolating fields for a given set of quantum numbers. For simplicity, as well as efficiency of the resulting nuclear interpolating fields, we will use a single interpolating field per baryon, selected to have good overlap with the single baryon ground state, as well as being comprised of a small number of quark level terms. The utility of the above form of the nuclear interpolating fields is twofold. Firstly, it allows us to derive the reduced weights we need for Eq. 2.2. Secondly, interpolating fields that are derived starting from Eq. 2.3 may have better overlap with the nuclear ground states as it is well-known that hadronic degrees of freedom provide a successful description of much of nuclear physics.

The calculation of the reduced weights, $\tilde{W}_{h}^{\left(b_{1}, b_{2} \cdots b_{A}\right)}$, in the hadronic interpolating field is straightforward. It amounts to combining individual hadrons of given quantum numbers to build a multi-hadron state of definite parity, angular momentum, isospin, and strangeness. This construction can be readily automated and can be performed recursively using the known Clebsch-Gordan coefficients of $\mathrm{SU}(2)$ for both the spin and isospin (or $\mathrm{SU}(3)$ flavour if so desired).

We have written a $\mathrm{C}++$ symbolic manipulation program that generates the hadronic reduced weights using the above approach. In Ref. [8], we have used this to produce a complete basis of orthonormal interpolating fields with spatial wave-functions restricted to a single point for all 
nuclei up to $A=4$ and have also constructed a selection of states for $A>4$. Generically for larger $A$, more complicated spatial wave-functions are required because of the Pauli exclusion principle, resulting in an exponential growth of the number of possible interpolating fields as $A$ increases (this reflects the problem faced in nuclear structure calculations as $A$ becomes large).

The reduced weights of the quark interpolating fields of Eq. 2.2 can be calculated by equating the two forms of the nuclear interpolating fields as described in Ref. [10].

\section{Multi-baryon contractions}

In this section, we consider how the interpolating fields presented in the previous section can be used to generate the correlation functions of multi-baryon systems. A general multi-hadron two point function is given by

$$
\left\langle\mathscr{N}_{1}^{h}(t) \overline{\mathscr{N}}_{2}^{h}(0)\right\rangle=\frac{1}{\mathscr{Z}} \int \mathscr{D} \mathscr{U} \mathscr{D} q \mathscr{D} \bar{q} \mathscr{N}_{1}^{h}(t) \overline{\mathscr{T}}_{2}^{h}(0) e^{-\mathscr{S}_{Q C D},}
$$

where $\mathscr{S}_{Q C D}$ and $\mathscr{Z}$ are the QCD action and partition function respectively, and $\mathscr{D} \mathscr{U}, \mathscr{D} q \mathscr{D} \bar{q}$ are the gluon and quark field integration measures respectively. We have also introduced explicit dependence of the interpolating fields on the Euclidean time separation, $t$, and consider a two point function with different creation and annihilation interpolating fields with commensurate quantum numbers. For a given choice of the interpolating fields, it is straightforward to perform the Grassmann integral over the quark fields and re-write the correlation function in terms of the quark propagators. However, for an efficient calculation of the two point function we need to be mindful of the structure of the interpolating fields.

One successful class of interpolating fields for two or more hadron systems is one in which a plane wave basis at the level of the hadronic interpolating fields is used. This amounts to projecting the individual hadrons comprising the multi-body system to definite momentum states, while preserving the spatial transformation properties of the overall multi-hadron system. In this case, the complexity of the spatial wave-function is such that the number of terms contributing to Eq. 2.2 is rather large and hadronic interpolating fields have to be used in order to build the desired two point function. Using this type of interpolating fields at the sink while using simple spatial wave functions for the source allows for efficient computations of correlation functions for nuclei up to atomic number $A=5$. These methods were used in the recent NPLQCD work that can be found in Ref. [12, 13]. For more details of how this approach is implemented we refer to [10].

Here we will focus on a method appropriate to atomic numbers, $A \geq 5$. It is straightforward to see that by making use of Wick's theorem [14], the numerator of Eq. 3.1 performing only the integration over quark fields takes the form

$$
\begin{aligned}
{\left[\mathscr{N}_{1}^{h}(t) \overline{\mathscr{N}}_{2}^{h}(0)\right]_{U}=} & e^{-\mathscr{S}_{e f f}[U]} \sum_{k^{\prime}=1}^{N_{w}^{\prime}} \sum_{k=1}^{N_{w}} \tilde{w}_{h}^{\prime\left(a_{1}^{\prime}, a_{2}^{\prime} \cdots a_{n_{q}}^{\prime}\right), k^{\prime}} \tilde{w}_{h}^{\left(a_{1}, a_{2} \cdots a_{n_{q}}\right), k} \times \\
& \sum_{\mathbf{j}} \sum_{\mathbf{i}} \varepsilon^{j_{1}, j_{2}, \cdots, j_{n_{q}}} \varepsilon^{i_{1}, i_{2}, \cdots, i_{n_{q}}} S\left(a_{j_{1}}^{\prime} ; a_{i_{1}}\right) S\left(a_{j_{2}}^{\prime} ; a_{i_{2}}\right) \cdots S\left(a_{j_{n_{q}}}^{\prime} ; a_{i_{n_{q}}}\right),
\end{aligned}
$$

where the primed and unprimed indices are associated with the sink and source interpolating fields, respectively and are composite colour, spinor, flavour and spatial indices and $[\ldots]_{U}$ indicates the 
value of the enclosed expression on a fixed gauge field. In addition, $S_{e f f}[U]$ denotes the pure gauge part of the QCD action together with the logarithm of the determinant of the Dirac matrix and $S\left(a^{\prime} ; a\right)$ denotes the quark propagator. The above expression of Wick's theorem, can be written in terms of the determinant of a matrix $G$ whose matrix elements are given by

$$
G\left(\mathbf{a}^{\prime} ; \mathbf{a}\right)_{j, i}= \begin{cases}S\left(a_{j}^{\prime} ; a_{i}\right) & \text { for } a_{j}^{\prime} \in \mathbf{a}^{\prime} \text { and } a_{i} \in \mathbf{a} \\ \delta_{a_{j}^{\prime}, a_{i}} & \text { otherwise }\end{cases}
$$

where, as before, $\mathbf{a}^{\prime}=\left(a_{1}^{\prime}, a_{2}^{\prime} \cdots a_{n_{q}}^{\prime}\right)$ and $\mathbf{a}=\left(a_{1}, a_{2} \cdots a_{n_{q}}\right)$. Note also that the non-trivial block of the matrix $G\left(\mathbf{a}^{\prime} ; \mathbf{a}\right)$ is of size $n_{q} \times n_{q}$, hence for computing its determinant we only need to consider this block. For this reason, in the following discussion, the matrix $G\left(\mathbf{a}^{\prime} ; \mathbf{a}\right)$ denotes only this small non-trivial block.

Making use of this definition, the full nuclear correlation function can be written as

$$
\left\langle\mathscr{N}_{1}^{h}(t) \overline{\mathscr{N}}_{2}^{h}(0)\right\rangle=\frac{1}{\mathscr{Z}} \int \mathscr{D} \mathscr{U} e^{-\mathscr{S}_{e f f}} \sum_{k^{\prime}=1}^{N_{w}^{\prime}} \sum_{k=1}^{N_{w}} \tilde{w}_{h}^{\left(a_{1}^{\prime}, a_{2}^{\prime} \cdots a_{n q}^{\prime}\right), k^{\prime}} \tilde{w}_{h}^{\left(a_{1}, a_{2} \cdots a_{n_{q}}\right), k} \times \operatorname{det} G\left(\mathbf{a}^{\prime} ; \mathbf{a}\right) .
$$

The determinant of a matrix of size $n_{q}$ can be evaluated in $n_{q}^{3}$ operations (for example via LU decomposition) instead of the naive $n_{q}$ ! operations, so making use of this representation of the nuclear correlation function is numerically advantageous. Furthermore, because of the flavourblindness of the strong interaction, the matrix $G\left(\mathbf{a}^{\prime} ; \mathbf{a}\right)$ is block diagonal, as a result the determinant calculation breaks into a product of smaller determinants, one for each flavour.

Given the reduced weights determined above and appropriate quark propagators, the implementation of Eq. 3.4 is very fast, scaling polynomially with the number of terms in the source and sink quark level interpolating fields as well as the number of quarks per flavour. The total cost of this form of contractions scales as $n_{u}^{3} n_{d}^{3} n_{s}^{3} \times N_{w}^{\prime} N_{w}$, where $N_{w}^{\prime} N_{w}$ are the number of terms in the sink and source quark interpolating fields respectively. ${ }^{2}$ As a result, if we can construct interpolating fields with sufficiently small number of terms, correlation functions with a very large atomic number $A$ can be constructed.

\section{Nuclear correlation functions}

We have performed preliminary studies to investigate the numerical efficiency of these methods. Results for the quark-hadron approach have been presented in Ref. [8] and here we focus on the determinant-based approach. Calculations are performed on an ensemble of gauge configurations generated with a tadpole-improved Lüscher-Weisz gauge action and a clover fermion action with tadpole-improved tree-level clover coefficient. The gauge links entering the fermion action are stout smeared, with $\rho=0.125$. Three flavours of quarks with masses corresponding to the physical strange quark mass were used. The lattice spacing, $a \sim 0.145 \mathrm{fm}$, and the dimensions of the lattice are $L^{3} \times T=32^{3} \times 48$ corresponding to a physical volume of $(4.6 \mathrm{fm})^{3} \times 7.0 \mathrm{fm}$. We have performed a large number of measurements from spatially distinct sources on an ensemble

\footnotetext{
${ }^{2}$ The expectation of polynomial scaling of contractions was noted by D. B. Kaplan in Ref. [15]. However, the scaling of $N_{w}$ and $N_{w}^{\prime}$ with the atomic number $A$ is generically exponential.
} 

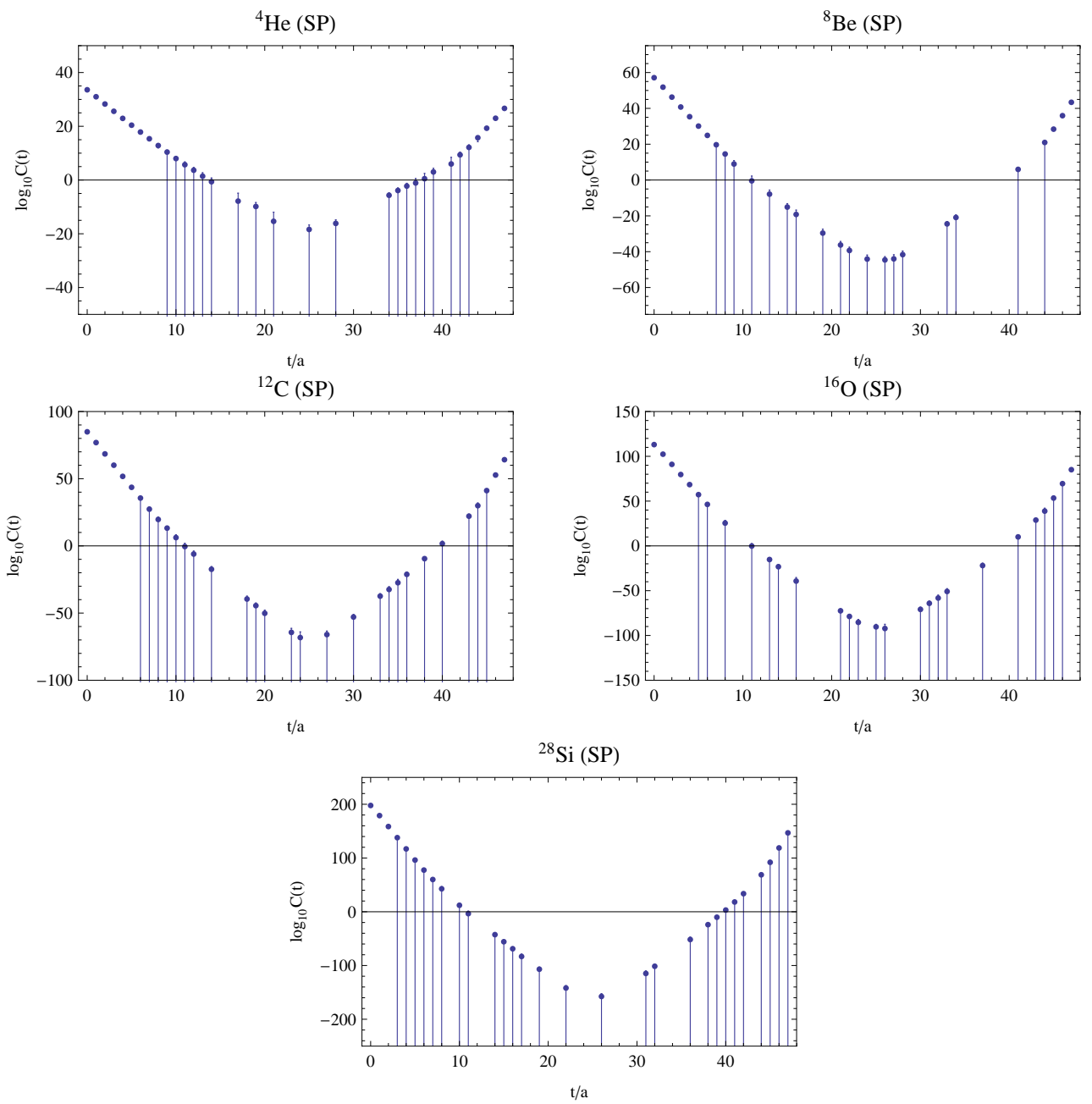

Figure 1: Correlation functions for nuclear systems, ${ }^{4} \mathrm{He},{ }^{8} \mathrm{Be},{ }^{12} \mathrm{C},{ }^{16} \mathrm{O}$ and ${ }^{28} \mathrm{Si}$.

of a $\mathscr{O}(250)$ gauge configurations well separated in HMC evolution time. All calculations are performed in double precision and care is taken to preserve the dynamic range of correlation functions by rescaling quark propagators before contractions are performed.

In Fig. 1, the logarithms of correlation functions are shown for correlators with the quantum numbers of the light nuclei, ${ }^{4} \mathrm{He},{ }^{8} \mathrm{Be},{ }^{12} \mathrm{C},{ }^{16} \mathrm{O}$ and ${ }^{28} \mathrm{Si}$. Error bars that reach the lower axis of the plots indicate that the correlator has fluctuations that are negative at one standard deviation. The extracted energies for each of the atomic number $A<20$ systems are consistent with a system of $A$ nucleons but with large uncertainties at present (for ${ }^{28} \mathrm{Si}$, no flattening of the effective mass is seen before the signal is lost). Given the large number of near-threshold energy levels expected in these complex nuclear systems (see Ref. [8] for an example for $A=4$ ), a clean extraction of the ground state binding energies of these systems is beyond the current work. In addition, the baryon number density of the larger systems $\left(0.3 \mathrm{fm}^{-3}\right.$ for $\left.{ }^{28} \mathrm{Si}\right)$ is substantial and volume effects are expected to be significant. It will be necessary to use larger volumes, increase greatly the statistical precision and improve the interpolating operators that we have used in order to obtain the binding energies and excitation spectra of these systems. Nevertheless, this study demonstrates the computational 
feasibility of lattice QCD calculations of light nuclei.

\section{Conclusions and outlook}

In this talk, we have discussed a systematic way of constructing interpolating fields for multibaryon systems and, have investigated methods of performing the Wick contractions with these interpolating fields in lattice QCD. We have found approaches that enable the calculation of two point functions for systems with large atomic number A, in a computationally feasible manner. In addition, we have demonstrated their effectiveness by calculating correlators with baryon number up to $A=28$. Given the expected finite volume spectra of such complex systems, significant advances are required in order to extract ground state energies from these correlators. Finally, the methods described here may prove useful in calculations of QCD at non-zero baryon density, where projection on to a given baryon number is required.

\section{References}

[1] S.R. Beane et al. (NPLQCD Collaboration), Phys.Rev. D80, 074501 (2009), 0905.0466

[2] T. Yamazaki, Y. Kuramashi, A. Ukawa (PACS-CS Collaboration), Phys.Rev. D81, 111504 (2010), 0912.1383

[3] S. Beane et al. (NPLQCD Collaboration), Phys.Rev.Lett. 106, 162001 (2011), 1012.3812

[4] S. Beane et al. (NPLQCD Collaboration), Mod.Phys.Lett. A26, 2587 (2011), 1103.2821

[5] T. Inoue (for HAL QCD Collaboration) (2011), 1111.5098

[6] S. Beane et al. (NPLQCD Collaboration), Phys.Rev. D85, 054511 (2012), 1109.2889

[7] T. Yamazaki, Y. Kuramashi, A. Ukawa, Phys.Rev. D84, 054506 (2011), 1105.1418

[8] S. Beane et al. (NPLQCD Collaboration) (2012), 1206.5219

[9] T. Doi, M.G. Endres (2012), 1205.0585

[10] W. Detmold, K. Orginos (2012), 1207.1452

[11] R. Feynman, M. Kislinger, F. Ravndal, Phys.Rev. D3, 2706 (1971)

[12] K. Orginos, ”Algorithm Developments for Nuclear LQCD” (2011), talk presented at Extreme Computing and its Implications for the Nuclear Physics/Applied Mathematics/Computer Science Interface, INT, July 6th 2011

[13] S. Beane, E. Chang, S. Cohen, W. Detmold, H. Lin et al. (2012), 1206.5219

[14] G. Wick, Phys.Rev. 80, 268 (1950)

[15] D.B. Kaplan, ”The Old Fart Talk” (2007), talk presented at Domain Wall Fermions at Ten Years, BNL, March 17th 2007 